\title{
ON LINEAR MODELS WITH RATIONAL EXPECTATIONS WHICH ADMIT A UNIQUE SOLUTION
}

\author{
L. BROZE and A. SZAFARZ* \\ Free University of Brussels, 1050 Brussels, Belgium \\ Received December 1982, final version received September 1983
}

Based on a generalization of Doob's theorem, the method used in this paper is applied to derive the unique reduced form of a general linear model containing rational expectations of the
current endogenous variables made in several previous periods. In this procedure there is no need for assumptions on the structure of the policy instruments.

\section{Introduction}

The derivation of reduced forms of rational expectations models is much discussed in the economic literature. Many authors [e.g. Blanchard (1979), Gourieroux et al. (1982), McCallum (1981), Shiller (1978) and Taylor (1977)] have been confronted with the presence of an infinity of solutions when models contain expectations of future endogenous variables. In a recent paper Broze et al. (1982) develop a method based on a generalization of Doob's theorem which permits to derive the reduced form for a large class of models of this kind.

Models without expectations of future variables are also of interest. In this case it is established that, except in some degenerate cases, the reduced form is unique. Various solution techniques have been suggested [Aoki and Canzoneri (1979) and Visco (1981)]. Visco (1981) solves a general linear model containing expectations of the present endogenous variables made in several previous periods, but he supposes from the start that the policy instrument vector $x$ has a known feedback structure.

In this paper we also consider a general model but, using the mathematical background developed in Broze et al. (1982), we need no particular assumption on the structure of the stochastic process $x$. However, unlike to what happens when models contain expectations of future variables, the martingales we introduce at the beginning of the procedure disappear when the final unique reduced form is established. Moreover, we show that the solution obtained by Visco is a particular case of ours in which the 'feedback assumption' is made.

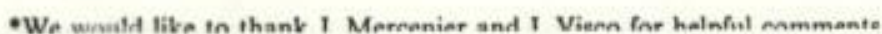


The solution technique applied in this paper allows to introduce the assumption on the structure of the policy instrument at the very end of the procedure, i.e., after the reduced form of the model has been established. Therefore it becomes possible to simulate the effects of various policies (simple or multiple feedback with different parameters, exogenous policies, etc.). In section 2, we give the expression of the reduced form written as a sum of the 'perfect foresight' solution of the model and a 'stochastic' term. This expression is valid for any locally integrable vector $x$.

\section{The model}

We consider the following general model: ${ }^{1}$

$$
y_{t}=\sum_{k=1}^{R} B_{k} y_{t \mid t-k}+\sum_{i=1}^{q} A_{i} y_{t-i}+C x_{t}+u_{t}
$$

where:

(1) The information available at time $t$ is given by the set of the present and past values of the vector processes $y$ and $x$,

$$
I_{t}=\left(y_{t}, y_{t-1}, \ldots, x_{t}, x_{t-1}, \ldots\right)
$$

We will call $f$ the increasing sequence of $\sigma$-algebras $\left(I_{t}\right)$.

(2) The expectations are 'rational', i.e.,

$$
y_{t \mid t-k}=\mathrm{E}\left[y_{t} \mid I_{t-k}\right] .
$$

(3) The processes $y=\left(y_{t}\right)$ and $x=\left(x_{t}\right)$ are integrable and the perturbation process $u=\left(u_{t}\right)$ satisfies the usual assumptions,

$$
\mathrm{E}\left[u_{t}\right]=0, \quad \forall t \quad \text { and } \mathrm{E}\left[u_{t} \mid I_{t-k}\right]=0, \quad \forall t, \forall k>0 .
$$

$y$ is the vector of endogenous variables (noted $z$ by Visco) and $x$ can denote a vector of either policy instruments or exogenous variables; the only requirement on $x$ is the integrability, i.e., the existence of a finite $E\left[x_{t}\right]$ for all $t$. Note that the weaker assumption of local integrability, i.e., the existence of a finite $\mathrm{E}\left[\mid x_{t} \| I_{t-1}\right]$ for all $t$, would be sufficient for further developments.

A generalized Doob's theorem [see e.g. Broze et al. (1982)] shows that,

'This model is the same as Visco's (1981, p. 356) general model but the lagged values of $y=\left(y_{1}\right)$ appear explicitly. Moreover, when $B_{2}=\ldots=B_{p}=0$, it can be solved easily by a projection technique [see Chow (1980, p. 48)].

for any integrable and adapted process $g=\left(g_{t}\right)_{1 \in \mathrm{N}}$ there exist a martingale $M=\left(M_{t}\right)_{t \in N}$ and an integrable predetermined process $P=\left(P_{t}\right)_{t \in N}$ such that, for any $t \in N, g_{t}=M_{t}+P_{t}$. We apply this theorem to the $(p+1)$ vector processes defined by $\left(y_{t}\right),\left(\mathrm{E}\left[y_{t+1} \mid I_{t}\right]\right), \ldots,\left(\mathrm{E}\left[y_{t+p} \mid I_{t}\right]\right)$, which are obviously integrable and adapted to $\mathscr{g}$

$$
\begin{aligned}
& y_{t}=M_{t}^{0}+P_{t}^{0}, \\
& \mathrm{E}\left[y_{t+1} \mid I_{t}\right]=M_{t}^{1}+P_{t}^{1}, \\
& \vdots \\
& \mathrm{E}\left[y_{t+p} \mid I_{t}\right]=M_{t}^{p}+P_{t}^{p},
\end{aligned}
$$

where $M^{0}, M^{1}, \ldots, M^{p}$ are martingales and $P^{0}, P^{1}, \ldots, P^{p}$ are integrable predetermined processes.

Proposition. If $M^{0}, M^{1}, \ldots, M^{p}$ and $P^{0}, P^{1}, \ldots, P^{p}$ are the processes defined by (2.2), then for any $k \in\{1, \ldots, p\}$

$$
M_{t}^{k}+P_{t}^{k}=y_{t+k}+\sum_{j=0}^{k-1}\left(M_{t+k-1-j}^{j}-M_{t+k-j}^{j}\right) \text { w.p. } 1^{2}
$$

This proposition will be established in the appendix by proving two preliminary lemmas.

Using (2.2) and (2.3), (2.1) becomes

$$
\begin{aligned}
V_{p} y_{t}= & \sum_{k=1}^{2} B_{k} \sum_{j=0}^{k-1}\left(M_{t-j-1}^{j}-M_{t-j}^{j}\right)+\sum_{i=1}^{q} A_{i} y_{t-j} \\
& +C x_{t}+u_{t} \\
= & \sum_{j=0}^{p-1} W_{j+1}\left(M_{t-j-1}^{j}-M_{t-j}^{j}\right)+\sum_{i=1}^{q} A_{i} y_{t-i}+C x_{t}+u_{t},
\end{aligned}
$$

where

$$
\begin{aligned}
& V_{0}=I, \\
& V_{j}=I-\sum_{k=1}^{L} B_{k} \text { for } j \in\{1, \ldots, p\}, \\
& W_{j}=\sum_{k=j}^{p} B_{k} .
\end{aligned}
$$


- Degenerate cases appear when at least one $V_{j}$ is singular. In these cases, when some necessary conditions on the structure of $x$ are fulfilled, there exists an infinite set of reduced forms for (2.1). Otherwise (2.1) has no solution.

Let us consider the non-degenerate case. To eliminate the martingales in (2.4) we project (2.1) successively on $I_{t-1}, I_{t-2}, \ldots, I_{t-p}$ and, by (2.2), obtain for $j \in\{1, \ldots, p\}^{3}$

$$
\begin{aligned}
V_{j}\left(M_{t-j}^{j}+P_{t-j}^{j}\right)= & \sum_{k=j+1}^{p} B_{k}\left(M_{t-k}^{k}+P_{t-k}^{k}\right)+\sum_{i=j}^{q} A_{i} y_{t-i} \\
& +\sum_{i=1}^{j-1} A_{i}\left(M_{t-j}^{j-j}+P_{t-j}^{j-j}\right)+C \mathrm{E}\left[x_{t} \mid I_{t-j}\right] .
\end{aligned}
$$

We subtract each of these projections from the preceding one and use Lemma 1 (see the appendix) and (2.2),

$$
\begin{aligned}
V_{j}\left(M_{i-j}^{j}-M_{t-j-1}^{j}\right)= & \sum_{i=1}^{L} A_{i}\left(M_{i-j}^{j-j}-M_{i-j-1}^{j-j}\right) \\
& +C\left(\mathrm{E}\left[x_{t} \mid I_{t-j}\right]-\mathrm{E}\left[x_{t} \mid I_{t-j-1}\right]\right),
\end{aligned}
$$

or

$$
V_{j}\left(M_{i-j}^{j}-M_{t-j-1}^{j}\right)=K_{j}\left(M_{t-j}^{0}-M_{t-j-1}^{0}\right)+\sum_{i=1}^{L} L_{i, j} C w_{t-j+1, i}
$$

where the matrices $K_{j}$ and $L_{i, j}$ are determined recursively by

$$
\begin{aligned}
& K_{0}=I, \\
& K_{j}=A_{j}+\sum_{i=1}^{j-1} A_{j-i} V_{i}^{-1} K_{i}, \\
& L_{j, j}=I, \\
& L_{i, j}=\sum_{k=1}^{j-1} A_{k} V_{j-k}^{-1} L_{i, j-k} \text { for } i<j,
\end{aligned}
$$

and

$$
w_{t, s}=\mathrm{E}\left[x_{t} \mid I_{t-s}\right]-\mathrm{E}\left[x_{t} \mid I_{t-s-1}\right] .
$$

Using (2.1) and (2.2) we write

$$
M_{t-j}^{0}-M_{t-j-1}^{0}=u_{t-j}+C w_{t-j, 0}
$$

and substituting first $(2.12)$ in (2.8) and then (2.8) in (2.4) we obtain the unique reduced form of $(2.1),{ }^{4}$

$$
\begin{aligned}
y_{t}= & \sum_{i=1}^{q} V_{p}^{-1} A_{i} y_{t-i}+V_{p}^{-1} C x_{i}+V_{p}^{-1} u_{t} \\
& -\sum_{j=0}^{p-1} V_{p}^{-1} W_{j+1} V_{j}^{-1}\left(K_{j} C w_{t-j, 0}+K_{j} u_{t-j}+\sum_{i=1}^{j} L_{i, j} C w_{t-j+i, i}\right) .
\end{aligned}
$$

It is then possible to separate (2.13) into the reduced form of the 'perfect foresight version' of the model and the so-called 'stochastic part' $\Phi_{t}^{p}$,

$$
y_{t}=\sum_{i=1}^{q} V_{p}^{-1} A_{i} y_{t-i}+V_{p}^{-1} C x_{t}+\Phi_{t}^{p} .
$$

$\Phi_{i}^{p}$ describes a serially correlated error structure. ${ }^{5}$ Moreover, the influence of the policy instruments $x$ in the 'stochastic part' of the reduced form of $(2.1)$ is completely included in the bivariate stochastic process $w$ which denotes differences between successive expectations of the same value of the policy vector.

\section{Visco's model}

The model considered by Visco (1981) is

$$
y_{t}=A_{1} y_{t-1}+\sum_{i=1}^{p} B_{i} y_{t \mid t-i}+C x_{t}+u_{i}
$$

${ }^{4}$ It is clearly possible to eliminate the lagged values of $y$ in (2.13) and express $y_{1}$ (for $t \geq q$ ) given $q$ initial conditions $y_{0}, \ldots, y_{q}-1$,

$$
\begin{aligned}
& y_{t}=V_{j}^{-1} \sum_{i=1}^{0} F_{t-1} y_{q-1}-\sum_{i=0}^{t} \sum_{j=0}^{n-1} V_{j}^{-1} W_{j+1} V_{j}^{-1} K_{j} C_{i}\left(C w_{t-1-1,0}\right. \\
& \left.+u_{t-j-k}\right)-\sum_{k=0}^{1-g} \sum_{j=0}^{0-1} \sum_{i=0}^{1} V_{j}^{-1} W_{j+1} V_{j}^{-1} L_{i, j} C_{k} C_{w_{t-j+i-1, i}} \\
& +\sum_{k=0}^{1-n} V_{0}^{-1} C_{k}\left(C x_{1-1}+u_{1-2}\right)
\end{aligned}
$$

The coefficients $C_{2}$ and $F_{4-1}$ are appropriately determined in terms of the coefficients of the model (i.e., $B_{k}, A_{i}, C$ ).

'If $q<p$ we suppose that $A_{i}$ is zero for $i>q$. This is of course a non-restrictive assumption. 
with the 'feedback assumption' on $x$,

$$
x_{t}=G_{t} y_{t-1}+v_{t}
$$

where $\left(G_{t}\right)$ is a deterministic sequence included in the information and $v=\left(v_{t}\right)$ is a perturbation.

We solve model (3.1) by the previous technique, without any assumption on $x$. We have

$$
\begin{aligned}
& K_{1}=A_{1}, \\
& K_{j}=A_{1} V_{j-1}^{-1} K_{j-1} \text { for } j>1, \\
& L_{j, j}=I, \\
& L_{i, j}=A_{1} V_{j-1}^{-1} L_{i, j-1} \text { for } i<j, \\
& w_{t-j, 0}=v_{t-j}, \\
& w_{t-j+i, i}=G_{t-j+i}\left(M_{t-j}^{j-1}-M_{t-j-1}^{j-1}\right) .
\end{aligned}
$$

Introducing (3.2) in the reduced form, we obtain (after a lot of algebra) Visco's result, i.e.,

$$
y_{t}=V_{p}^{-1} A_{1} y_{t-1}+V_{p}^{-1} C x_{t}+\Phi_{t}^{p},
$$

where

$$
\begin{aligned}
\Phi_{i}^{p}= & \sum_{j=0}^{p-1} V_{p}^{-1} W_{j+1} V_{j}^{-1} K_{j}\left(C v_{t-j}+u_{t-j}\right) \\
& +\sum_{j=1}^{p-1} \sum_{i=1}^{j} V_{p}^{-1} W_{j+1} V_{j}^{-1} L_{i, j} C W_{t-j+i, i}+V_{p}^{-1} u_{t} \\
= & u_{t}-V_{p}^{-1} W_{1} C v_{t}+\sum_{i=1}^{p-1} R_{t, i}\left(u_{t-i}+C v_{t-i}\right) .
\end{aligned}
$$

The values of the matrices $R_{t, i}$ are the same as Visco's $R_{t}^{l}$ (p. 359); they depend on the coefficients of the model (3.1) and on the deterministic part of the policy $G$.

\section{Concluding remarks}

The method used in this paper allows to express the reduced form of models with expectations of the current endogenous variables made in several previous periods. In Broze et al. (1982) models containing expectations of future endogenous variables are considered. More generally, the procedure using a generalization of Doob's theorem is applicable to any linear model containing rational expectations of the form $y_{t+x \mid t-i}(s \geqq 0, i>0)$. The number of arbitrary martingales which will appear in the reduced form will be the highest value of $s$.

This paper shows that Visco's results on model (3.1) and (3.2) can be generalized to model (2.1). The conclusion is then that for non-degenerate linear model with rational expectations the reduced form appears to be the sum of the solution of the 'perfect foresight version' of the model and a serially correlated error term in which the influence of the policy instruments can be described by perturbations due to differences between successive expectations of these instruments.

\section{Appendix: Proof of proposition (2.4)}

Two lemmas on the processes introduced in (2.2) are first established.

Lemma I. For any $k \in\{1, \ldots, p\}$,

$$
M_{t}^{k}+P_{t}^{k}=M_{t}^{k-1}+P_{t+1}^{k-1} .
$$

Proof. Using (2.2) and a property of conditional expectations, we obtain, successively,

$$
\begin{aligned}
M_{t}^{k}+P_{t}^{k} & =\mathrm{E}\left[y_{t+k} \mid I_{t}\right] \\
& =\mathrm{E}\left[\mathrm{E}\left[y_{t+k} \mid I_{t+1}\right] \mid I_{t}\right] \\
& =\mathrm{E}\left[M_{t+1}^{k-1}+P_{t+1}^{k-1} \mid I_{t}\right] .
\end{aligned}
$$

Since $M^{k-1}=\left(M_{t}^{k-1}\right)$ is a martingale and $P^{k-1}=\left(P_{t}^{k-1}\right)$ is a predetermined process, it follows that

$$
\begin{aligned}
& \mathrm{E}\left[M_{t+1}^{k-1} \mid I_{t}\right]=M_{t}^{k-1}, \\
& \mathrm{E}\left[P_{t+1}^{k-1} \mid I_{t}\right]=P_{t+1}^{k-1} .
\end{aligned}
$$

Introducing (A.3) and (A.4) in (A.2) we establish (A.1).

Lemma 2, For any $k \in\{1, \ldots ., p\}$. 


$$
P_{i}^{k}=P_{t+k}^{0}+\sum_{j=1}^{k}\left(M_{i+k-j}^{j-1}-M_{t+k-j}^{j}\right)
$$

Proof. (A.5) will be proved by induction. For $k=1$ we have by (A.1)

$$
P_{t}^{1}=P_{t+1}^{0}+\left(M_{t}^{0}-M_{i}^{1}\right) \text {. }
$$

Let us now suppose that

$$
P_{t}^{k-1}=P_{t+k-1}^{0}+\sum_{j=1}^{k-1}\left(M_{t+k-1-j}^{j-1}-M_{t+k-1-j}^{j}\right) .
$$

(A.1) implies that

$$
P_{t}^{k}=M_{t}^{k-1}-M_{t}^{k}+P_{t+1}^{k-1} .
$$

Then from (A.7) and (A.8),

$$
\begin{aligned}
P_{t}^{k} & =M_{t}^{k-1}-M_{t}^{k}+P_{t+k}^{0}+\sum_{j=1}^{k-1}\left(M_{i+k-j}^{j-1}-M_{t+k-j}^{j}\right) \\
& =P_{t+k}^{0}+\sum_{j=1}^{k}\left(M_{i+k-j}^{j-1}-M_{i+k-j}^{j}\right) . \square
\end{aligned}
$$

Proposition. For any $k \in\{1, \ldots, p\}$,

$$
M_{i}^{k}+P_{t}^{k}=y_{t+k}+\sum_{j=0}^{k-1}\left(M_{t+k-1-j}^{j}-M_{t+k-j}^{j}\right) \text {. }
$$

Proof. (A.5) implies that

$$
M_{t}^{k}+P_{t}^{k}=M_{i}^{k}+P_{t+k}^{0}+\sum_{j=1}^{k}\left(M_{i+k-j}^{j-1}-M_{t+k-j}^{j}\right) .
$$

By adding and subtracting $M_{t+k}^{0}$ in the right-hand side of (A.9),

$$
M_{t}^{k}+P_{t}^{k}=P_{t+k}^{0}+M_{t+k}^{0}+\sum_{j=0}^{k-1}\left(M_{t+k-1-j}^{j}-M_{t+k-j}^{j}\right) .
$$

Since

$$
P_{t+k}^{0}+M_{t+k}^{0}=y_{t+k}
$$

the result is proved.

\section{References}

Aoki, M. and M. Canzoneri, 1979, Reduced forms of rational expectations models, Quarterly Journal of Economics 93, 59-71.

Blanchard, O.J., 1979, Backward and forward solutions for economies with rational expectations, American Economic Review, Papers and Proceedings 69, 114-118

Broze, L., J. Janssen and A. Szafarz, 1982, On solutions of linear models with rational expectations, Discussion paper 8208 (C.E.M.E., Université Libre de Bruxelles, Brussels).

Chow, G.C., 1980, Econometric policy evaluation and optimization under rational expectations, Journal of Dynamics and Control 2, 47-59.

Gourieroux, C., J.J. Laffont and A. Monfort, 1982, Rational expectations in dynamic linear models: Analysis of the solutions, Econometrica $50,409-425$.

MacCallum, B.T, 1981, On non-uniqueness in rational expectations models: An attempt at perspective, NBER working paper 684

Shiller, R.J. 1978, Rational expectations and the dynamic structure of macroeconomic models: A critical review, Journal of Monetary Economics 4, 1-44.

Taylor, J.B., 1977, Conditions for unique solution in stochastic macroeconomic models with rational expectations, Econometrica 45, 1377-1385.

Visco, 1., 1981, On the derivation of reduced forms of rational expectations models, European Economic Review 16, 355-365. 